\title{
Erratum to: Persistence and dissipation of flubendiamide and its risk assessment on gherkin (Cucumis sativus $\mathrm{L}_{\text {.) }}$
}

\author{
M. Paramasivam • C. Selvi • S. Chandrasekaran
}

Published online: 21 April 2014

(C) Springer International Publishing Switzerland 2014

\section{Erratum to: Environ Monit Assess \\ DOI 10.1007/s10661-014-3745-2}

The original version of this article unfortunately contained a mistake.

In the article title and introduction section, gherkin (Cucumis anguria L.) should have been gherkin (Cucumis sativus L.). doi.org/10.1007/s10661-014-3745-2.

M. Paramasivam $(\bowtie) \cdot$ C. Selvi $\cdot$ S. Chandrasekaran

Pesticide Toxicology Laboratory, Department of Agricultural Entomology, Tamil Nadu Agricultural University,

Coimbatore, Tamil Nadu 641 003, India

e-mail:sivam25@gmail.com 